ORIGINAL ARTICLE

\title{
Procedural sedation in paediatric minor procedures: a prospective audit on ketamine use in the emergency department
}

\author{
D Y Ellis, H M Husain, J P Saetta, T Walker
}

Emerg Med J 2004;21:286-289. doi: 10.1136/emj.2003.007229

See end of article for authors' affiliations

.....................

Correspondence to: Mr J P Saetta, Emergency Department, Queen Elizabeth II Hospital, Welwyn Garden City Herffordshire AL7 $4 \mathrm{HQ}$, UK; john.saetta@nhs.net

Accepted for publication 15 October 2003

\begin{abstract}
Objectives: To further evaluate the safety profile and efficacy of intramuscular ketamine for procedural sedation during paediatric minor procedures in the emergency department and to ascertain parental satisfaction with the treatment of their children.

Methods: A prospective audit of ketamine use in a UK district general hospital involving 89 children requiring minor procedures. Children received topical anaesthesia followed by an intramuscular injection of ketamine $4 \mathrm{mg} / \mathrm{kg}$ and intramuscular atropine $0.02 \mathrm{mg} / \mathrm{kg}$. The procedure was assessed by way of a physician completed form and by evaluation of questionnaires given to parents to gauge levels of satisfaction.

Results: No child required admission to hospital and there were no serious complications. A high level of satisfaction was expressed by all the parents/guardians of the children treated.

Conclusions: High levels of satisfaction among parents and staff together with the avoidance of hospital admission and improved resource management should be a sufficient incentive for hospital trusts to consider the establishment of this type of service.
\end{abstract}

p is a common practice for children to undergo minor procedures in the emergency department including cleaning and closure of superficial lacerations, incision of abscesses, and extraction of foreign bodies. An important aspect of their treatment is the method used to reduce pain and anxiety during the procedure, which can cause more stress than the injury itself.

Some children, depending on age, may cooperate with their treatment under local anaesthetic. However, many young children are uncooperative despite attempts at distraction. It would appear that child restraint in the execution of minor procedures is commonplace in emergency departments in the United Kingdom. Parents also suffer if witnessing or participating in the restraint of their own child. The so called "minor" procedure results in psychological stress in the child, his parents, and care workers. The authors believe this is unnecessary and largely outdated.

If a child needs pharmacological sedation and anxiolysis then a general anaesthetic is often considered the gold standard in the hospital setting. However, in practice, it is not practical to provide this in a timely manner for all children. In most instances, the provision of an anaesthetic service for all children undergoing minor procedures places a heavy burden on theatre time, inpatient beds, and frequently results in unacceptable delays to treatment. In addition, general anaesthesia is not without risk.

It is therefore practical to provide some children with what is termed "procedural sedation". The American College of Emergency Physicians defines this as a method to "induce a state that allows the patient to tolerate unpleasant procedures while maintaining cardiorespiratory function independently and continuously." In our hospital, we use ketamine for procedural sedation.

Ketamine was first developed in 1962 and was used as a field anaesthetic by the United States (US) army in the Vietnam war. Its introduction as an intravenous anaesthetic in the hospital setting started in the 1970s. The early 1990s saw the first publications of its use in children by non-anaesthetists in emergency departments in the US. Its use now in the US is extensive and it remains popular in the developing world. ${ }^{1-4}$

Ketamine produces a combination of analgesia, amnesia, and sedation at subanaesthetic doses $(<6 \mathrm{mg} / \mathrm{kg}$ intramuscular (IM)), with minimal effect on the airway and vital reflexes. Unwanted side effects include nausea, vomiting, hypersalivation, and agitation/hallucinations, the last of which is reported less commonly in children than in adults. Airway problems have been reported although these have been almost invariably transient.

The emergency department at the QEII Hospital has in place a system permitting procedural sedation in children. The purpose of this paper is twofold: to report on a prospective audit of paediatric sedation using ketamine and to highlight the advantages of and the resources needed for running such a service in UK hospitals.

\section{METHODS}

A total of 89 children were involved in the audit over the period of a year. All cases were considered urgent but were not emergencies, and as such allowed for pre-planning and preparation for the procedure. The children were considered to require procedural sedation by a consultant, specialist registrar, or staff grade doctor and the sedation was carried out according to a strict protocol (available on the journal web site http://www.emjonline.com/supplemental). This protocol was modified after a previous retrospective study in $1997 / 1998^{5}$ and is based on the protocol used at the Loma Linda University, USA. ${ }^{1}$ Doctors completed a checklist/data recording form for each child recording all aspects of the procedure including timings, adequacy of sedation, and complications. The continuous presence of an advanced paediatric life support (APLS) trained person was mandatory.

A printed and verbal explanation of the procedure was provided and written consent obtained. Patients were starved for four hours before the procedure was undertaken, as per protocol. A patch of Emla (lidocaine 2.5\%, prilocaine 2.5\%) 
cream was applied to the intended site of injection at least one hour before the start of the procedure. Parental/carer satisfaction was assessed by means of a questionnaire that was taken home and posted back to the department up to two weeks later.

A dose of $4 \mathrm{mg} / \mathrm{kg}$ of ketamine and $0.02 \mathrm{mg} / \mathrm{kg}$ of atropine (standard paediatric dose, used as an antisialagogue) was both given IM in the same injection site. If sedation was deemed inadequate by the doctor carrying out the procedure, a booster dose of $2 \mathrm{mg} / \mathrm{kg}$ was given after 10 minutes. If appropriate, the wound site was infiltrated with a local anaesthetic (lidocaine, bupivacaine) before the procedure was started for additional analgesia and postoperative pain relief. Based on previous reports, intravenous cannulation was not routinely performed. ${ }^{1}$

Pulse oximetry, respiratory rate, pulse, and blood pressure were recorded as a baseline and every five minutes after injection of the drugs. Oxygen by facemask with a reservoir bag was only applied if the oxygen saturations decreased to less than $96 \%$.

After the procedure, the child was allowed to recover in a quiet area within the children's section of the emergency department. A qualified paediatric nurse was responsible for monitoring the patient during the procedure and throughout the recovery period.

\section{RESULTS ON PROCEDURAL ASPECTS}

Of the 89 cases audited 51 (57\%) were male and 38 (43\%) female. The age range was $1-10$ years old.

Eighty six (97\%) of the parents were given the ketamine information sheet and 58 (65\%) of the children had Emla cream applied although $10(17 \%)$ of these had the cream on for less than the recommended one hour.

All the children had at least respiratory rate or pulse oximetry recorded and $82(92 \%)$ of the children had all the relevant observations recorded (pulse, respiratory rate, blood pressure, and pulse oximetry). The lowest recorded oxygen saturations were $94 \%$.

Four $(4.5 \%)$ of the children required a second dose of ketamine, the maximum dose given being $6 \mathrm{mg} / \mathrm{kg}$. Adequate sedation was achieved in 10 minutes or less for 81 (91\%) children.

Local anaesthetic was injected after ketamine in 55 (62\%) of children. The vast majority of these children required suturing of a wound.

Table 1 shows the procedures done under ketamine.

Each procedure was completed in 15 minutes or less in 81 $(91 \%)$ of children with the longest procedure lasting 37 minutes.

Table 2 shows the side effects profile. There were no serious adverse effects reported in any of the children.

Mean recovery time was 101 minutes $(20-430)$ and all children were discharged home. Recovery time was defined as the time from procedure completion until the child

\begin{tabular}{ll} 
Table 1 & Procedures done under \\
\hline Procedure & Patients $(\mathbf{n})$ \\
\hline Suturing & $68(76)$ \\
Lips & 22 \\
Faces/foreheads & 18 \\
Finger tips/thumbs & 19 \\
Others & 9 \\
Foreign body removal & $14(16)$ \\
Incision and drainage of abscess & $5(6)$ \\
Excision of skin tag & $1(1)$ \\
Dressing & $1(1)$ \\
\hline Data in parentheses are percentages. & \\
\hline
\end{tabular}

fulfilled the discharge criteria in the protocol (as assessed by the nursing staff).

\section{RESULTS OF PARENT/CARER SATISFACTION SURVEY}

Survey forms were given to all parents and 78 (88\%) responded.

Seventy (96\%) parents said they were given an explanation about ketamine. Of these, $100 \%$ found the explanation easy to understand.

Sixty seven (86\%) stayed with the child through the procedure and six $(8 \%)$ reported problems. These problems usually centred on an initial concern the child was not adequately sedated when the doctor wanted to start the procedure and so further time was allowed before starting. The medical notes for these children indicated no concern on the part of the doctor. Two of the four children requiring second doses of ketamine had this recorded as a "problem" by the parent.

All parents/carers stayed with the child for recovery and nine $(12 \%)$ reported events during recovery. These events were more nebulous ranging from vomiting to noise in the department and slow recovery time.

None of the children required admission to hospital.

The side effects reported by parents within 48 hours after leaving the emergency department are included in table 2 and the time taken for the child to return to normal as defined by the parents at home can be seen in table 3. The remaining nine cases $(16 \%)$ all returned to normal within 72 hours except one case who took two weeks.

Nightmares were a feature in seven (9\%) cases but of these four $(4.5 \%)$ could not rule out having nightmares before their visit to the emergency department.

In answer to the satisfaction section of the questionnaire, $75(96 \%)$ of parents were happy with the treatment in the emergency department and 73 (94\%) would be happy to have ketamine treatment for their children again.

All the parents ticked the very satisfied/satisfied box.

\section{DISCUSSION}

Our experience with ketamine would support previous literature on the safe and effective use of this drug in the emergency department.

It ties in with the previous audit carried out at this hospital that, although done retrospectively, also showed no serious adverse events in 100 cases together with high parental satisfaction. ${ }^{5}$

In our series the majority of adverse effects from ketamine were minor and self limiting. The fact that we were able to discharge all our children home the same day was particularly relevant. Our mean recovery time is comparable with other studies but the very wide range of our recovery times perhaps points to a significant variation in the kinetics of ketamine between people. Certainly, the extreme times at both ends of the recovery range are exceptional and are, at least in part, attributable to the subjective nature of the assessment of young children. The parental questionnaire allowed us to assess side effects seen from the parents' perspective during the procedure and in the first 48 hours at home. None of these reported side effects caused excessive parental concern. It was also reassuring to see such a high percentage of children returning to "normal" within 24 hours, as assessed by the parents at home. It should be noted that the parental assessment of normal is reasonably subjective and may take into account recovery from the original injury and not just the sedation aspect. Other centres have also reported high parent satisfaction with IM ketamine. ${ }^{6}$

In our small series we did not witness any serious side effects but they can occur and such cases have been reported 
Table 2 Side effects of ketamine sedation

\begin{tabular}{|c|c|}
\hline Early & Late (within 48 hours) \\
\hline $\begin{array}{l}\text { During procedure } \\
\text { Vomiting* } 1(1) \\
\text { Othert } 1(1) \\
\text { During recovery } \\
\text { Vomiting* } 10 \text { (11) } \\
\text { Nausea } 2(2) \\
\text { Hallucinations } 2 \text { (2) } \\
\text { Agitation } 6 \text { (7) } \\
\text { Othert } 6 \text { (7) }\end{array}$ & $\begin{array}{l}\text { Drowsiness } 31(40) \\
\text { Pain at site of procedure } 20(26) \\
\text { Loss of coordination } 20(26) \\
\text { Nausea } 15 \text { (19) } \\
\text { Changes in sleep pattern } 14(18) \\
\text { Other unusual behaviour } 13(17) \\
\text { Agitation } 11 \text { (14) } \\
\text { Vomiting* } 10(13)\end{array}$ \\
\hline
\end{tabular}

in the literature. ${ }^{178}$ However, incidences of ketamine associated airway problems are not only uncommon but also usually transient, responding to repositioning of the head to open the airway. At the doses used for sedation, recorded cases of apnea all recovered uneventfully after between 5-40 seconds of bag-valve-mask ventilation. None of these cases required intubation and all recovered uneventfully thereafter. In fact, a review of 97 published reports of ketamine use revealed two cases of laryngospasm requiring intubation in healthy children from 11589 administrations. This is an incidence of less than $0.017 \%$. $^{3}$ The doses of ketamine used in these two cases were not made clear in the document.

Although serious side effects are rare, physicians should take steps to prevent or identify them early. The procedure should only be performed where suction, oxygen and resuscitation facilities are available. A paediatric nurse, with basic training in recovery techniques, should be present at all times to monitor the child and all participating doctors and nurses should be proficient in advanced airway management. There should be strict limitations on the dose of ketamine used as well as an option to perform the procedure under general anaesthetic should the sedation or procedure be unsuccessful.

Despite significant evidence on the safety of ketamine it seems that few emergency departments in the UK use this technique on a regular basis. The authors' experience is that in most hospitals resistance to ketamine use in the emergency department comes from the anaesthetic department.

Our own anaesthetic department was justifiably concerned about the prospect of ketamine being used in the emergency room in an uncontrolled environment and without appropriate supervision or knowledge of the drugs involved. It was therefore decided to liaise with the anaesthetic department from the very beginning to draw up guidelines that would permit the safe use of ketamine by non-anaesthetists in the emergency department.

In our view, any department wishing to start using ketamine sedation must have strict guidelines in place and will need to spend time and resources training staff, in

Table 3 Time taken for the child to return to normal after ketamine sedation

\begin{tabular}{ll}
\hline Time (h) & Patients (n) \\
\hline$<2$ & $19(26)$ \\
$2.1-6$ & $21(28)$ \\
$6.1-12$ & $14(19)$ \\
$12.1-24$ & $11(15)$ \\
$>24^{*}$ & $9(12)$ \\
\hline
\end{tabular}

*All the remainder were within 72 hours except one who took two weeks. This child had previously been diagnosed with "attention deficit hyperactivity disorder". Data in parentheses are percentages. particular paediatric nurses, in relevant aspects of care. An appropriate area should be available with a quiet section for recovery. Staffing must be adequate at times when ketamine is to be used. General guidelines for procedural sedation and analgesia in the emergency department are available.'

The data on the safety profile of ketamine are limited by the small sample size in our audit but combined with previous published work at this hospital the number of cases rises to 189. No serious adverse events were recorded in any of these cases and since the formal audit was stopped in February 2000 we have continued to use the ketamine data forms and have yet to record such an event. Thus, in total, our hospital has carried out well in excess of 250 ketamine sedations without a serious adverse event.

Extensive use in the USA has led to very large numbers of cases audited with similar results. In one review of 1022 cases no child required admission because of ketamine sedation and the authors concluded "ketamine [at this dose IM] is highly effective, has a wide margin of safety, does not require intravenous access, and uniquely preserves protective airway reflexes"

Sedative agents such as midazolam are already regularly used in the emergency room and other areas of the hospital without guidelines. There is no evidence that ketamine is any less safe than other commonly used sedatives, in fact the risks of airway compromise or cardiorespiratory instability may be less with ketamine. ${ }^{10}$

The combination of midazolam and ketamine to reduce some side effects has been investigated although the outcome is still unclear. ${ }^{11} 12$

The analgesic effect of ketamine is also significant when choosing appropriate sedation. A recent study found ketamine a safe and effective alternative to morphine in the immediate postoperative period. ${ }^{13}$

Introducing ketamine sedation to a UK district general hospital should have advantages for several departments. The experience gained in this department has opened up further possibilities including a reduction in operating lists and inpatient admissions. In addition, the authors feel it is appropriate for children with limb fractures requiring "manipulation under anaesthesia" to be included in a growing list of conditions that may be amenable to the procedural sedation provided by ketamine. Manipulations under anaesthesia already make up $20 \%$ of cases done under ketamine in some centres in the USA.

Our method of managing paediatric minor injuries seems to work well with high levels of satisfaction among healthcare workers and parents alike. With this technique we have been able to avoid some of the most frustrating aspects involved in the treatment of injured children such as overnight stays with prolonged fasting and cancellations secondary to "more urgent" cases taking limited theatre time. At the same time we have not compromised the safety of the children. 
Perhaps the way forward is a large multicentre study randomising children to either admission and general anaesthesia or ketamine sedation in the emergency department. Data from this, including parental satisfaction, child pain scoring, safety, and admission details may establish any potential for improving patient care and resource allocation.

\section{ACKNOWLEDGEMENTS}

The clinical audit department at the Queen Elizabeth II Hospital, especially Ann Ainsworth and Heather Davis.

\section{Contributors}

JPS initiated the audit and together with TM ascertained the procedural guidelines and assured appropriate training. HH supervised the audit and the collation of data. DYE did the literature research and wrote the original draft; DYE and TM modified this draft. All the authors contributed to the final draft.

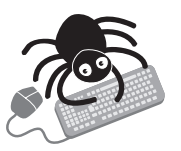

The protocol for the use of intramuscular ketamine for children is available on the journal web site (http:// www.emjonline.com/supplemental).

\section{Authors' affiliations}

D Y Ellis, H M Husain, J P Saetta, Department of Emergency Medicine, Queen Elizabeth II Hospital, Welwyn Garden City, Hertfordshire, UK

T Walker, Department of Anaesthesia, Queen Elizabeth II Hospital

Funding: none.

Conflicts of interest: none declared.

\section{REFERENCES}

1 Green SM, Rothrock SG, Lynch EL, et al. Intramuscular ketamine sedation in the emergency department: safety profile in 1,022 cases. Ann Emerg Med 1998;31:688-97.

2 Green S. Ketamine sedation for paediatric procedures: part 1, a prospective series. Ann Emerg Med 1990;19:1024-32.

3 Green SM, Johnson NE. Ketamine sedation for paediatric procedures: part 2, review and implications. Ann Emerg Med 1990;19:1033-46.

4 Green SM, Clem KJ, Rothrock SG. Ketamine safety profile in the developing world: survey of practitioners. Acad Emerg Med 1996;3:598-604.

5 Holloway VJ, Husain HM, Saetta JP, et al. Accident and emergency department led implementation of ketamine sedation in paediatric practice and parental response. J Accid Emerg Med 2000;17:25-8.

6 McGlone RG, Ranasinghe S, Durham S. An alternative to "brutacaine": a comparison of low dose intramuscular ketamine with intranasal midazolam in children before suturing. $J$ Accid Emerg Med 1998;15:231-6.

7 Smith JA, Santer $\amalg$. Respiratory arrest following intramuscular ketamine injection in a 4-year old child. Ann Emerg Med 1993;22:613-15.

8 Mitchell RK, Koury SI, Stone CK. Respiratory arrest after intramuscular ketamine in a 2-year old child. Am J Emerg Med 1996;14:580-1.

9 American College of Emergency Physicians. Clinical policy procedural sedation and analgesia in the emergency department. Ann Emerg Med 1998;31:663-77.

10 Drummond GB. Comparison of sedation with midazolam and ketamine: effects on airway muscle activity. Br J Anaesth 1996;76:663-7.

11 Wathen JE, Roback MG, Mackenzie T, et al. Does midazolam alter the clinical effects of intravenous ketamine sedation in children? A double-blind, randomized, controlled, emergency department trial. Ann Emerg Med 2000;36:579-88.

12 Funk W, Jakob W, Riedl T, et al. Oral preanaesthetic medication for children: double-blind randomized study of a combination of midazolam and ketamine vs midazolam or ketamine alone. Br J Anaesth 2000;84:335-40.

13 Aspinall RL, Mayor A. A prospective randomized controlled study of the efficacy of ketamine for postoperative pain relief in children after adenotonsillectomy. Paediatr Anaesth 2001;11:333-6. 